\title{
DESENVOLVIMENTO DE SISTEMA SISMOGRÁFICO DE ALTA RESOLUÇĀO
}

\author{
F.Taioli
}

PALAVRAS-CHAVE: sismógrafo, acelerômetro, alta resolução.

TAIOLI, F. (1995) Desenvolvimento de sistema sismográfico de alta resolução. Bol. IG-USP, Sér. Clent., 26:29-43.

\section{RESUMO}

Este trabalho apresenta o projeto, desenvolvimento e testes de um sistema sismo. gráfico para utilização em trabalhos de sismica aplicada à engenharia, hidrogeologia e meio ambiente, baseado na eletrôrica incorporada em um microcomputador tipo IBM-PC.

Desenvolveram-se, como sensores, acelerómetros piezoelétricos especiais para uso em sismografia, assim como amplificadores e filtros para atenderem às exigências impostas por aquelas aplicaç̋̄es. O interfaceamento com o microcomputador é feito por intermédio de uma placa de expansão conversora A/D (analógico-digital), e a velocidade necessária para a obtenção de altas frequêencias foi conseguida utilizando-se o DMA (direct memory access - acesso direto à memória), disponível no microcomputador tipo IBM-PC. Para isto, foi desenvolvido um programa de controle que, além de se utilizar đo DMA, emula as funçōes normais e o modo de operação de um sismógrafo digital convencional.

Foram efetuados diversos testes de laboratório em cada parte do sistema. $\mathrm{O}$ acelerômetro apresentou comportamento linear (dentro de $\pm 0,5 \mathrm{~dB}$, entre $10 \mathrm{~Hz}$ e $2000 \mathrm{~Hz}$ ). Os amplificadores e filtros acompanharam a mesma linearidade, entre $20 \mathrm{~Hz}$ e $1000 \mathrm{~Hz}$.

Diversos testes de campo têm mostrado a operacionalidade e aplicaçăo do sistema. Conclui-se que o sistema 6 factivel, a relativo baixo custo, $\mathrm{e}$ atende as necessidades básicas para os trabathos de sismica aplicada a engenharia, hidrogeologia e meio ambiente, sendo que a arquitetura adotada pode ser utilizada para outros instrumentos geofisicos.

\section{ABSTRACT}

This paper presents the design, development and tests of a prototype of a seismograph system for seismic studies applied to engineering, hydrogeology and environmental problems. The system is based on the in board electronic of an IBM-PC type microcomputer.

Piezoelectric accelerometers, as well as the amplifiers and filters were specially designed to be used in seismography. The interfacing with the microcomputer is accomplished by an A/D (analog to digital) converter board. The acquisition velocity to record high frequencies was obtained by using the DMA (direct memory access) available in the microcomputer. Control software was developed to allow the use of the DMA and to simulate the normal functions of a standard digital seismograph.

In laboratory tests the accelerometers showed a linear behavior $( \pm 0.5 \mathrm{~dB}$, between $10 \mathrm{~Hz}$ and $2000 \mathrm{~Hz}$ ), and the amplifiers and filters exhibit the same linearity between $20 \mathrm{~Hz}$ and $1000 \mathrm{~Hz}$. Field tests have shown the system to be operational.

Thus, the system proposed and developed is feasible, relatively cheap, and suitable

Departamento de Geologia Econỏmica e Geofisica Aplicada, Instituto de Geociéncias/USP, São Paulo, Brasil. 
for the objectives of seismic work applied to engineering, hydrogeology and environmental problems. It is also suggested that the same architecture can be used in other geophysical instruments.

\section{INTRODUÇÃO}

Os métodos de prospecção sísmica foram desenvolvidos no final do século passado e início do presente, sendo hoje técnicas consagradas na exploraçăo mineral e de hidrocarbonetos.

Os princípios básicos do método não serâo aqui abordados, uma vez que podem ser encontrados em extensa bibliografia (e.g. Jakoski, 1950; Seguin, 1971; Anstey, 1979; Born, 1978; Coffen, 1978; Sheriff, 1978; Al-Sadi, 1980; Dobrin, 1981; Neidell, 1982; Parasnis, 1986; Sharma, 1986; Robinson \& Coruh, 1988; Sheriff, 1989; Telford et al., 1990, entre outros).

Se, por um lado, a literatura abordando a metodologia sísmica é abundante, não é possível dizer o mesmo a respeito de equipamentos sismográficos. A razão disto talvez seja a falta de interesse dos fabricantes em divulgar os progressos técnicos no detalhe que um artigo cientifico exige.

Encontra-se algumas referências sobre a utilização do método sísmico no final do século passado, porém, este método só veio a se desenvolver após o surgimento do amplificador a válvulas, que ocorreu em torno de 1925 (Born, 1960). Keppner (1991) atribui a Ludger Mintrop (1880-1956) a primeira utilização de sísmica com sucesso na definição de reserva petrolifera, que teria ocorrido em 1925. Nessa época foi possivel desenvolver uma instrumentaçâo adequada, utilizando sensores elétricos, amplificador a válvulas e sistema de registro por galvanômetros e película fotográfica. A partir dai, grandes aperfeiçoamentos foram feitos mas esta tecnologia básica perdurou até meados da década de 60 . Todos estes equipamentos eram de registro analógico, e o aperfeiçoamento ocorreu na direção de minia- turizar as válvulas e posteriormente substitui-las por transistores. Os primeiros instrumentos operavam com dois canais, porém em 1940 o padrâo da indústria já era a utilização de 12 canais, sendo que logo $\mathrm{em}$ seguida passaram a operar com 24 canais.

Durante a Segunda Guerra Mundial, um rápido progresso ocorreu na tecnologia de registro em fitas magnéticas, de forma que no final da década de 40 algumas companhias dispunham de equipamento com registro magnético para uso próprio, porém o primeiro modelo disponivel comercialmente surgiu em 1954. Sem dúvida, este foi um aprimoramento notável, pois permitia o armazenamento e reprodução dos registros sísmicos. A introduçāo de registros magnéticos e dos transistores também permitiu o surgimento de outras técnicas de geração de frentes de ondas sísmicas. Algumas delas se baseavam na queda de um peso no terreno. Apesar da energia liberada dessa forma ser menor do que se utilizando dinamite, tornou-se possivel fazer o stacking ou somatória de vários registros do mesmo ponto, tendose, desta forma, amplificação dos sinais coerentes e atenuação dos ruídos aleatórios. Essas técnicas passaram a ser utilizadas em regiōes onde o uso de dinamite era impossivel, por razőes de segurança.

Na década de 50 também foi introduzido o processamento de dados por computador, que abreviou o tempo de tratamento dos dados, dinamizando também a interpretação dos dados. A introdução do método vibroseis foi, sem dúvida, inovação revolucionária $\mathrm{e}$ digna de nota. Este método utiliza-se de uma fonte de energia de freqüência variável ao longo do tempo, ou seja, não se trata 
de uma fonte impulsiva. O sinal recebido pelo geofone é registrado, e então é efetuada uma correlação cruzada com o sinal emitido pelos vibradores. O resultado final é um registro igual àquele gerado por uma fonte impulsiva.

No entanto, a maior mudança tecnológica nos instrumentos geofísicos, em geral, e nos sísmicos, em particular, ocorreu em 1963, com o surgimento de instrumentação digital, onde o registro dos sinais não é mais feito de maneira contínua, mas de forma discreta (numérica). Isto trouxe maior faixa dinâmica aos instrumentos e maior capacidade de processamento, uma vez que o registro efetuado no campo já tinha o formato necessário para sua utilização nos computadores. Evidentemente, isso foi possivel graças ao contínuo aumento da escala de integração de componentes e à disponibilidade no mercado de conversores analógico-digitais rápidos, precisos e confiáveis.

No início dos anos 70 , graças à disponibilidade de instrumentos com grande número de canais $(96)$ e à grande capacidade de processamento dos novos computadores, iniciaram-se os levantamentos em 3D (três dimensões), que trouxcram um poder maior à capacidade de interpretação dos dados.

Por volta de 1975 surgiram também os sismógrafos de exploração telemétricos, onde a informação sísmica digitalizada é enviada via rádio, para a unidade central de registro. Isto tomou possivel a execução de levantamentos sísmicos em regiōes antes inacessíveis.

$\mathrm{Na}$ década de 80 , devido ao grande progresso na tecnologia de semicondutores, foi possível o desenvolvimento de sistemas sismográficos com maior resposta em freqüência. Iniciouse, então, a utilização da técnica sísmica para trabalhos onde uma alta resolução (Knapp \& Steeples, 1986a, b) era desejada (c.g., levantamentos em regiōes produtoras de carvão).
Atualmente, um sistema bastante difundido é o de aquisição descentralizada, que permite operar com grande número de canais (eventualmente mais de 5.000 canais) usando-se cabos sismográficos de fibra óptica para o envio das informações sísmicas, já digitalizadas, à unidade de registro.

Todos estes desenvolvimentos citados tiveram aplicaçăo generalizada na indústria de prospecção de hidrocarbonetos. Porém, hoje em dia, estão disponiveis sistemas sismográficos para outras aplicações, tais como, hidrogeologia, geologia de engenharia, etc. Tais sistemas têm incorporado portas para conexão com microcomputadores tipo PC, e podem gravar arquivos em discos magnéticos com formatação compatível. Os sismógrafos mais recentes trazem incorporados um microcomputador que gerencia todas as funções do sismógrafo, além de gravar os dados e eventualmente processá-los.

Os progressos com relação aos sensores (geofones) utilizados em prospecção também têm sido enormes ao longo do tempo. Os primeiros sensores consistiam em uma bobina móvel suspensa por molas em torno de um núcleo magnético, exatamente o mesmo principio usado atualmente, mas a grande diferença é que pesavam em torno de 5 a $6 \mathrm{~kg}$ (10 Hz de freqüência natural), e possuíam amortecimento de óleo (Bom, 1960). Com a adoção de técnicas de exploração mais complexas (e.g., CDP e filtros espaciais) e com o acréscimo do número de canais por instrumento, surgiu a necessidade de miniaturização dos geofones, o que foi possível com a introdução de ímãs de campos mais intensos e de amortecimento elétrico (resistivo). $\mathrm{Na}$ década de 40 , o aprimoramento da qualidade das válvulas disponiveis no mercado também permitiu o trabalho com sensores menos sensíveis, e portanto menores, sem comprometimento da qualidade final do registro. 
Com a introdução da tecnologia digital e o aumento acentuado da faixa dinâmica dos instrumentos, foi necessário novamente melhorar a qualidade dos sensores, que se conseguiu utilizando-se ímãs de ligas especiais.

$O$ incremento na utilização de levantamentos sísmicos de alta resolução levou os fabricantes a desenvolverem e produzirem também sensores com resposta em frequência mais ampla, visto que os convencionais são utilizáveis somente até $500 \mathrm{~Hz}$ (Washburn \& Wiley, 1941; Fail et al., 1962; Lamer, 1970; Lepper, 1981), apesar de serem lineares em frequências mais baixas (Lepper, 1981).

Klassen \& Van Peppen (1983) desenvolveram um geofone eletromagnético mais apropriado para trabalhos de resoluçāo maior, valendo-se da sensibilidade à aceleração do sensor $\mathrm{e}$ de um circuito eletrônico interno, no corpo do geofone. Segundo os autores, estes geofones mantêm sua linearidade até a freqüência de $500 \mathrm{~Hz}$.

Apesar das limitações, os sensores eletromagnéticos são ainda hoje os mais comumente utilizados pela sua versatilidade, portabilidade, bom desempenho, robustez e relativo baixo custo.

Paralelamente ao desenvolvimento e aprimoramento dos sensores eletromagnéticos, os transdutores piezoelétricos também apresentaram acentuado aperfeiçoamento, tornando-se pequenos e robustos graças à utilização de pastilhas sintéticas de propriedades piezoelétricas acentuadas. Esses sensores são mais apropriados a trabalhos com alta freqüência (Lepper, 1981) e trazem também a vantagem de operarem omnidirecionalmente. No entanto, o seu alto custo tem limitado sua aplicação a projetos especiais, quando o registro de alta freqưência é imperioso.

Em resumo, apesar das poucas referências bibliográficas a respeito dos desenvolvimentos tecnológicos aplica- dos a sismógrafos de exploração, tais como os trabalhos de Born (1960), Wolff \& Mercanti (1974) e de Keppner (1991), torna-se possivel identificar alguns dos grandes marcos tecnológicos, que trouxeram progressos notáveis à instrumentaçāo geofisica, em geral, e à sísmica, em particular. O primeiro ocorreu por volta de 1920 , com a utilização de amplificadores a válvula nos instrumentos de campo. O segundo deu-se após a Segunda Guerra Mundial, com a introdução dos registradores magnéticos e transistores. Um terceiro grande marco verificou-se na década de 60 , com a introdução de técnicas digitais de aquisiçăo de dados e processamento por computador. $O$ mais recente foi, sem dúvida, o surgimento do microcomputador pessoal, possibilitando o processamento de dados de maneira iterativa e, algumas vezes, integrado no sistema de campo.

\section{OBJETIVOS DO TRABALHO}

Com o decréscimo dos preços dos equipamentos e sistemas de processamento sismográficos, abriu-se uma grande possibilidade de utilização de métodos sísmicos em projetos de pequena envergadura (quando comparados à prospecção de hidrocarbonetos), tanto no setor mineral, como no apoio a obras de engenharia civil, de minas e problemas ambientais. Estas aplicações são, muitas vezes, detalhamentos de partes dos maciços, necessários para uma melhor compreensão do seu comportamento, frente às imposiçôes de novos regimes de distribuição de tensôes provocadas pela obra. Eventualmente, este tipo de levantamento necessita de modificaçôes instrumentais de relativa complexidade, praticamente impossivel em sistemas "fechados", adquiridos no mercado.

Um sistema sismográfico que se utiliza de toda a eletrônica embarcada 
em um microcomputador $\mathrm{PC}$, de arquitetura aberta, torna-se a opção natural de desenvolvimento. Acrescentado a isto, a utilização de acelerômetros piezoelétricos especialmente desenvolvidos para a faixa de freqüências de interesse no trabalho sismográfico, abre a possibilidade de se trabalhar com freqüencias mais elevadas, e conseqülentemente com maior resoluçāo, além de permitir a miniaturização de ferramentas para ensaios entre furos (crosshole), tomográficos e mesmo de perfilagem sônica, minimizando custos.

A arquitetura aberta de um sistema deste tipo permite, ainda, o futuro desenvolvimento de sensores e de fontes especiais para aplicações especificas, muitas vezes necessárias em trabalhos de detalhamento.

Portanto, o objetivo principal deste trabalho foi o de demonstrar a viabilidade de desenvolvimento de um sistema sismográfico baseado, primariamente, em um microcomputador PC, operando com acelerômetros como sensores.

\section{ARQUTTETURA DO SISTEMA}

A arquitetura do sistema desenvolvido segue sugestōes apresentadas em Anstey (1979) e Pieuchot (1984), além de detalhada análise de catálogos de equipamentos existentes no mercado, Esta arquitetura consiste em sensores (acelerômetros piezoelétricos) especialmente desenvolvidos para uso sismográfico, amplificadores e filtros ativos e conversor analógico-digital (facilmente encontrado no mercado nacional $\mathrm{e}$ internacional), compativel ao microcomputador PC, ao qual ele é conectado. Todo o comando das operaçōes é executado por um programa de controle especialmente desenvolvido para esse fim, e emula todas as operaçōes que normalmente são executadas pelos sismógrafos digitais convencionais. A seguir é discutido cada um dos componentes do sistema.

\section{Sensor}

Os sensores normalmente utilizados em trabalhos sismográficos (geofones) consistem em sistemas eletromagnéticos, onde uma bobina suspensa por meio de molas é colocada em tomo de um núcleo magnético fixo. O movimento entre estes dois elementos, a bobina e o imã, gera uma corrente na bobina proporcional à velocidade relativa. A resposta em freqüência deste tipo de sensor é função da constante elástica das molas e da massa da bobina, e a amplitude do sinal de saida é funçảo das características da bobina e das grandezas magnéticas do imã. Sua fabricação toma-se dificil devido à necessidade de materiais especiais e de molas que apresentem grande estabilidade, sensibilidade e robustez.

O acelerômetro convencional consiste em um sistema onde um cristal com propriedades piezoelétricas é colocado sobre uma base rigida, e sobre o cristal é assentada uma massa denominada massa sísmica. $\mathrm{O}$ movimento do conjunto faz com que o cristal gere uma corrente proporcional à sua aceleração. A resposta em frequêtncia deste tipo de sensor é dependente das massas sísmica e do cristal (quanto menores as massas sísmicas, maior a freqüência de ressonância), enquanto a amplitude do sinal também depende das propriedades piezoelétricas do cristal, da sua massa e da massa sísmica (quanto maiores as massas, maior o sinal). Portanto, torna-se necessária a compatibilização entre os tamanhos do cristal e da massa sísmica, com a faixa de freqüências desejada, e com o nivel de amplitude do sinal esperado.

Um problema a ser considerado relativo à utilização de acelerômetros piezoelétricos diz respeito à impedância extremamente elevada do cristal, o que impossibilita a transmissão do sinal gerado a longas distâncias.

O sensor desenvolvido neste tra- 
balho é um acelerômetro piezoelétrico formado por uma base de latão sobre a qual é colada uma pastilha de material piezoelétrico (zirconato titanato de chumbo e bário). Sobre a pastilha é colada uma massa sísmica também de latão, de diâmetro igual porém tendo o dobro da altura da pastilha. Os terminais elétricos são o próprio corpo da base (terminal negativo), e a massa sismica (terminal positivo). A fim de possibilitar a utilização de cabos sismográficos longos, colocou-se um pré-amplificador intemo, dentro do corpo do acelerômetro, fixado na base por meio de parafusos. Este pré-amplificador tem a funçâo de reduzir a impedância do sinal de saida e possibilitar o ajuste de ganho, de forma a permitir um intercâmbio dos acelerômetros no sistema. O ganho utilizado na calibração dos acelerômetros foi ajustado por meio de um potenciômetro, até se atingir um sinal de saída de 1 Volt/g $( \pm 1 \%)$ a $300 \mathrm{~Hz}$ em cada acelerômetro, onde g é a aceleraçăo da gravidade. A alimentação do pré-amplificador é fornecida por meio do cabo sismográfico. O esquema deste acelerômetro é apresentado na Figura 1. A Figura 2 mostra o diagrama elétrico esquemático do pré-amplificador utilizado internamente no acelerômetro. A teoria de funcionamento e cálculo das caracteristicas do acelerômetro podem ser encontradas em detalhes em Serridge \& Licht (1986).

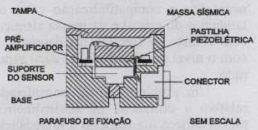

Figura 1 - Diagrama esquemático do acelerômetro piezoelétrico desenvolvido.

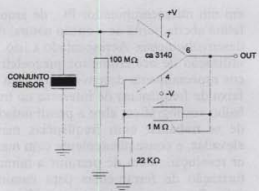

Figura 2 - Diagrama elétrico do circuito do préamplificador do acelerómetro desenvolvido.

O levantamento das características dinâmicas do acelerômetro foi efetuado neste projeto, utilizando a configuração experimental, conforme esquematizado na Figura 3, até a definição de uma boa solução mecânica e eletrônica. Em seguida foi efetuado um ensaio comparativo com padrões internacionais, para cada exemplar do acelerômetro construido. Os resultados obtidos estão apresentados nas Figuras 4 e 5 .

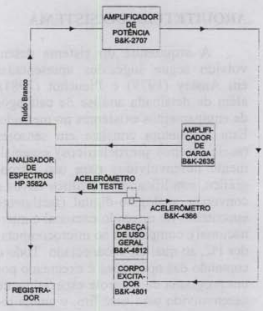

Figura 3 - Configuraçăo experimental utilizada para teste das caracteristicas dinâmicas dos acelerômetros. 


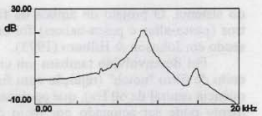

Figura 4 - Resposta em frequência de DC a 20 $\mathrm{kHz}$ do acelerómetro desenvolvido.

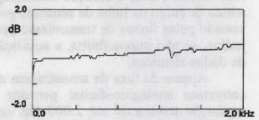

Figura 5 - Resposta em freqüência de DC a 2 $\mathrm{kHz}$ do acelerômetro desenvolvido.

A análise destes resultados permite concluir que o acelerômetro apresenta uma resposta plana, dentro de \pm 1 dB, na faixa de $10 \mathrm{~Hz}$ a $2.000 \mathrm{~Hz}$. O sinal de saída é da ordem de $1 \mathrm{Volt} / \mathrm{g}$. Medidas efetuadas em laboratório determinaram que o ruido instrumental destes sensores encontra-se na faixa de $10^{-3} \mathrm{~g}$; para uma freqüência de $200 \mathrm{~Hz}$, isto equivale a cerca de $600 \times 10^{-12} \mathrm{~m}$ de deslocamento das partículas, valor inferior ao menor deslocamento possível (aproximadamente $10 \times 10^{-9} \mathrm{~m}$ ) de ser captado pelos geofones eletromagnéticos encontrados no mercado (GeoSpace, 1995).

Tais características foram consideradas aceitáveis para o trabalho em sismografia de exploração. Foram construidos 14 acelerômetros no total, obedecendo sempre à mesma metodologia de calibração e levantamento das características dinâmicas.

\section{Conversor analógico-digital}

As características do conversor analógico-digital condiciona o projeto de todos os outros componentes do sistema, pois o desenvolvimento das demais partes depende de suas características de entrada e da possibilidade de programaçāo de suas interfaces. Diversos conversores $\mathrm{A} / \mathrm{D}$ foram pesquisados $\mathrm{e}$ considerados, tendo-se optado pelo conversor Lynx modelo $12 / 36$, que consiste em uma placa de expansão, compativel com os microcomputadores $\mathrm{PC}$, e disponivel no mercado nacional. Trata-se de um conversor de 12 bits (4096 niveis de digitalização), que possui uma série de características que o torna particularmente apropriado para o trabalho em questão. Estas características são:

a) velocidade de conversão de até 60.000 amostras por segundo;

b) entrada analógica multiplexada para até 16 canais independentes;

c) base de tempo interna $(2,00$ $\mathrm{MHz}$ ), independente da do microcomputador;

d) possibilidade de selecionar, via programa de controle, entre quatro niveis de amplificação, independentemente para cada canal;

e) possibilidade de inicialização das conversōes por sinal de sincronismo gerado externamente;

f) capacidade de acessar a memória do microcomputador independentemente de seu processador, por meio do DMA, (direct memory access - acesso direto à memória). Esta característica permite a coleta de dados na freqüência máxima de conversão, independente da velocidade da unidade central de processamento do microcomputador;

g) possibilidade de ser programado de maneira convencional, tanto em linguagens de baixo (Assembler) como de alto nível (Pascal, C e até mesmo Basic);

h) impedância da entrada dos amplificadores de $100 \mathrm{kOhm}$;

i) precisão de $\pm 1 / 2$ bit menos significativo;

j) as alimentações necessárias são 
fornecidas pelo próprio microcomputador;

1) possibilidade de operar em temperaturas entre $0^{\circ}$ e $50^{\circ} \mathrm{C}$.

Considerando-se que o padrão da indústria sismográfica utiliza o número de canais múltiplo de 6 , optou-se pelo desenvolvimento de um sistema com 12 canais, o que atende a este padrão, e também à grande parte dos trabalhos referidos anteriormente. A implementação de 12 canais permite a utilização desta placa a uma taxa de amostragem de 4.000 amostras por segundo por canal, que possibilita, de acordo com o teorema de amostragem, trabalhar com uma frequiência do sinal sísmico de até $2.000 \mathrm{~Hz}$.

\section{Amplificadores e filtros}

Foi desenvolvido especialmente um conjunto de amplificadores e filtros ativos cuja função principal é tornar os niveis dos sinais provenientes do sensor compativeis com as entradas do conversor analógico-digital. Para isto, é dado um ganho fixo de 100 vezes, o que equivale a $40 \mathrm{~dB}$. Um conjunto de filtros associados aos amplificadores, limita a banda de frequêencia entre $20 \mathrm{~Hz}$ e $1000 \mathrm{~Hz}$ ou $200 \mathrm{~Hz}$ e $1.000 \mathrm{~Hz}$.

$\mathrm{Na}$ implementação final, os filtros passa-altas são do tipo Chebyshev, que aplicam uma atenuação de $12 \mathrm{~dB} /$ oitava, e cujas frequências de corte ( 20 ou 200 $\mathrm{Hz}$ ) são selecionadas por jumper na placa de circuito impresso. Este estágio de filtros tem um ganho de $20 \mathrm{~dB}$, acima da frequéencia de corte.

O filtro passa-baixas é do tipo Chebyshev, implementado de forma a atenuar o sinal de $24 \mathrm{~dB} /$ oitava, e tendo freqüência de corte de $1.000 \mathrm{~Hz}$. Este filtro possui a rampa de corte mais abrupta, pois ele tem também a função de filtro antialias. Este estágio amplifica o sinal de 10 vezes, que, associado ao anterior, dá o ganho total de 100 vezes para a faixa de frequieencias de operação do sistema. O projeto de ambos os filtros (passa-altas e passa-baixas) foi baseado em Johnson \& Hilburn (1975).

Foi desenvolvido também um circuito de filtro "notch" (rejeição com frequêência central de $60 \mathrm{~Hz}$ ), que opcionalmente pode ser acionado por meio de um jumper Texas Instruments (1992). Este filtro adquire importância maior quando se utiliza o equipamento em áreas urbanas, onde a indução eletromagnética de ruído na linha de sensores provocado pelas linhas de transmissão prejudica, se nāo impossibilita, a aquisição de dados sísmicos.

Apesar da taxa de amostragem do conversor analógico-digital permitir a aquisição teórica de até $2.000 \mathrm{~Hz}$, optou-se pelo filtro de $1.000 \mathrm{~Hz}$ de rampa de corte menos abrupta, por razôes de custo.

Os amplificadores e filtros estão montados em placa de circuito impresso de tal maneira que se tem uma placa por canal e, por serem idênticas, são intercambiáveis. As doze placas foram condicionadas em uma caixa tipo maleta, com blindagem eletrostática de aluminio, e contendo ainda duas baterias seladas de 12 Volts e $6,5 \mathrm{Ah}$, que alimentam uma fonte estabilizada simétrica ( +8 e $-8 \mathrm{VDC})$, utilizada para alimentação dos sensores e dos circuitos de amplificação e filtros. O valor de tensâo de 8 Volts foi escolhido pois permite que se trabalhe, sem problemas, com tensão mínima das baterias de 10,5 Volts.

Depois da calibração de cada placa (obtida pelos métodos convencionais de laboratório, com sinais senoidais, e com ruído branco contínuo de amplitudes conhecidas) procurou-se efetuar teste que simulasse realmente as condições de campo, ou seja, o comportamento dos amplificadores e filtros quando solicitados a sinais transientes. Para isto, foi montado um circuito gerador de um pulso único com amplitude de aproximadamente $70 \mathrm{mV}$ e duração menor do 
que um ciclo de amostragem do conversor $\mathrm{A} / \mathrm{D}$ (menor do que $15 \mathrm{~ms}$ ). Este pulso foi injetado em cada um dos canais e a resposta foi registrada em arquivos no microcomputador, posteriormente recuperados para cálculo do espectro de freqüência contido no sinal. O resultado proveniente de tal análise corresponde à resposta dinâmica dos amplificadores e filtros, uma vez que o conteúdo em freqüências de um pulso é infinito. A Figura 6 traz o esquema da montagem experimental utilizada, e os gráficos obtidos, para as diversas combinações de filtros possiveis, estão apresentados nas Figuras 7 e 8 . A análise e comparação dos diversos gráficos permitem afirmar que os amplificadores e filtros apresentaram resposta em freqüência de acordo com o projeto, e comportamento bastante homogêneo (dentro de $\pm 5 \%$ ) entre todos os canais.

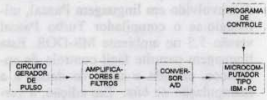

Figura 6 - Esquema instrumental utilizado para teste dinâmico dos filtros $\mathrm{e}$ amplificadores desenvolvidos.

Outra série de testes foi efetuada com uma configuração experimental, conforme mostrado na Figura 9. Os testes foram realizados por meio de um ruido branco injetado na entrada do amplificador de potência do sistema vibratório e observada a transformada de Fourier do sinal de saida, de tal forma que se obtinha, de maneira rápida, a resposta em frequêencia do circuito de amplificadores e filtros quando operando em conjunto com os acelerômetros. Foram efetuados ensaios deste tipo para as várias combinações possíveis de filtros, os resultados típicos são apresentados

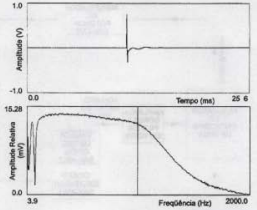

Figura 7 - Resposta dinâmica dos filtros de 20 $\mathrm{Hz}$, rejeição, $1.000 \mathrm{~Hz}$ e amplificadores associados.

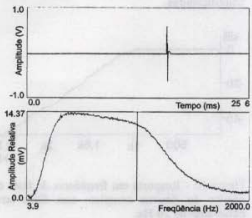

Figura 8 - Resposta dinâmica dos filtros de 200 $\mathrm{Hz}, 1.000 \mathrm{~Hz}$ e amplificadores associados.

nas Figuras 10 e 11. Pelos resultados destes ensaios, pode-se notar que as respostas obtidas são bastante semelhantes às dos amplificadores isolados.

\section{Cabo sismográfico}

Para a conexăo dos sensores aos respectivos circuitos de amplificação e filtros foi desenvolvido um cabo sismográfico especial, a partir de um condutor múltiplo, utilizado normalmente em telefonia, e portanto facilmente encontrado no mercado, composto por 22 fios flexiveis (AWG \# 24), com malha de cobre estanhado, que funciona como 


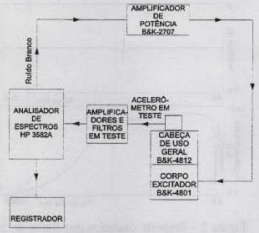

Figura 9 - Configuração experimental utilizada para testes do sistema acelerômetros, filtros e amplificadores.

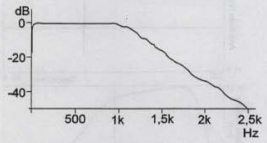

Figura 10 - Resposta em frequência de teste dinámico do sistema completo com filtro ativo passa-altas de $20 \mathrm{~Hz}$.

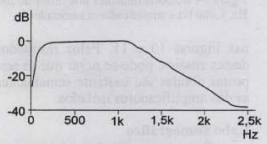

Figura 11 - Resposta em frequência de teste dinámico do sistema completo com filtro ativo passa-altas de $200 \mathrm{~Hz}$.

blindagem. Trabalhos anteriores executados (Taioli \& Ribeiro, 1990a, b), usando o mesmo cabo em outros sistemas de aquisição de dados de campo, mostraram resistência e durabilidade em condições adversas, qualidades estas imprescindiveis para os trabalhos sismográficos. As tomadas para cada sensor foram executadas por meio de uma incisão e ligaçăo de um outro cabo flexível com quatro condutores e comprimento de $0,30 \mathrm{~m}$, em cuja extremidade foi instalado um conector de quatro pinos que fornece a alimentação elétrica ao sensor e recebe os sinais sísmicos. As saidas estão espaçadas a cada 5 metros, de tal forma que o cabo tem um comprimento total de 60 metros. Em uma extremidade do cabo sismográfico foi instalado um conector de 24 pinos, do qual são utilizados 15 pinos (12 para os sinais sísmicos e 3 para polarizaçāo dos pré-amplificadores) para a ligação do cabo à caixa de amplificadores.

\section{Programa de controle}

O programa para executar o controle da operação de todo o sistema foi desenvolvido em linguagem Pascal, utilizando-se o compilador Turbo Pascal versão 5.5 no ambiente MS-DOS. Esta linguagem permite fácil controle de endereços, entrada e saída de dados e a manipulação de bits. Em adição, a versão utilizada do compilador Turbo Pascal possui uma biblioteca de comandos gráficos bastante poderosa, e uma linguagem estruturada que facilita a alteração do programa.

O programa de controle foi desenvolvido utilizando-se uma arquitetura que procura simular a operação dos sismógrafos digitais convencionais, a fim de reduzir o tempo de aprendizagem e a possibilidade de erro do operador. Ele pode ser descrito de dois modos diferentes: um é como o usuário o visualiza, enquanto o outro relaciona-se com o funcionamento interno do programa.

Para o usuário, após inicialização do programa, é apresentado um menu com quatro opçōes, as quais são:

a. ajusta parâmetros globais;

b. recupera arquivo, 
c. adquire novo registro, e

d. termina.

A primeira opção (a) permite ao usuário fornecer dados importantes, que seråo gravados no cabeçalho do arquivo de dados. Estas informações consistem em: título do trabalho que está sendo executado, tempo de atraso entre o gatilhamento e o início do registro, filtro passa-altas escolhido, offset utilizado (distância entre o ponto de tiro e o primeiro geofone da linha), espaçamento entre geofones, posiçāo do ponto de tiro na linha sísmica, direcão do tiro (direto, central ou reverso), seleção dos ganhos dos 12 canais, freqüência de amostragem, seleção do diretório de trabalho para armazenamento dos arquivos de dados e, finalmente, possibilidade de retornar ao menu inicial.

A segunda opção (recupera arquivo) permite recuperar um arquivo de um registro efetuado anteriormente e o apresenta no vídeo de forma gráfica. Convém salientar que esta opção não permite acesso ao registro, ou seja, não é possível alterá-lo ou utilizá-lo em operação de empilhamento, portanto o registro é preservado na sua forma original. Por outro lado, é apresentado um menu de opções que permite amplificar os tracos, aplicar um zoom e imprimir a tela. As outras opções disponíveis são a de adquirir um novo registro, verificar o ruído na linha sismica e retornar ao menu inicial.

A opção seguinte possibilita acesso ao modo de aquisição de novos registros e, conseqüientemente, à opção utilizada durante o trabalho normal, e que será detalhada adiante.

A última opção (termina), como o próprio nome diz, encerra o processamento e retorna o computador ao modo de processamento normal.

Em cada uma das opções escolhidas, o usuário tem a seu dispor um novo menu de opções que 0 orienta quanto às possibilidades do sistema.
Internamente, o programa é formado por diversos módulos que são acionados de acordo com as opções escoIhidas pelo usuário. Alguns módulos, entretanto, passam despercebidos, pois são os que acionam os endereços dos dispositivos de entrada e saída do conversor $\mathrm{A} / \mathrm{D}$, acionam o DMA, criam matrizes de dados, zeram matrizes de dados e fornecem informações especificas do equipamento para serem gravadas no cabeçalho do arquivo.

Os módulos de acesso disponivel ao usuário são descritos a seguir:

a) ruído na linha - mostra um gráfico de barras com a amplitude do sinal presente em cada um dos canais;

b) adquire registro - limpa o conteúdo da matriz de dados e a prepara para receber um novo bloco de dados, que será iniciado em sincronismo com o sinal de disparo proveniente da fonte sísmica;

c) grava arquivo - permite gravar no disco o conteúdo da matriz de dados, assim como o seu cabeçalho. Antes da gravação é solicitado um nome para o arquivo;

d) amplificar traços - permite aplicar um fator de multiplicação aos dados em uso. Esta opção não modifica os dados originais, pois é aplicado o fator de multiplicação simplesmente para tornar o registro mais visivel no monitor;

e) zoom - mostra somente a primeira metade dos dados, porém com maior detalhe. Isto objetiva facilitar a visualização da parte inicial do registro;

f) imprime tela - coloca disponível a uma impressora o conteúdo da região de dados do monitor. É fornecido também um pequeno cabeçalho para permitir sua identificação;

g) somar registro - cria uma segunda matriz de dados, inicialmente com o conteúdo da matriz de dados original, e armazena a somatória dos registros ponto por ponto. Uma vez esco- 
lhida esta opção, os próximos menus serão diferentes, pois permitirão, além das demais opções, efetuar somas positivas ou negativas;

h) menu inicial - esta opção permite o retorno ao menu inicial. Quando isto é feito, as matrizes de dados são zeradas $\mathrm{e}$ as variáveis do programa são levadas a valores predefinidos (default).

A opção "adquire novo registro" passa primeiramente pelo gráfico de ruido presente na linha, onde pode-se retornar ao menu anterior ou adquirir o registro. Caso se confirme a opção de adquirir registro, a tela do monitor é limpa e surge a mensagem "aguardando o trigger", significando que o sistema está inteiramente pronto para receber os dados, após o envio do sinal de sincronismo proveniente do sistema gerador das ondas seja enviado. Uma vez adquiridos os dados e armazenados na matriz de dados via DMA, ela é lida e, por meio de uma rotina gráfica, é colocada no monitor de video. Tendo em vista que o registro tem um comprimento de 1024 amostras e os monitores de video mais comuns não dispõem dessa resolução na horizontal, é apresentada somente metade dos dados (as amostras impares), porém o suficiente para que se tenha uma boa visualização do registro. Caso se opte pelo zoom, são apresentadas as primeiras 512 amostras, obtendo-se assim uma definição total da primeira metade do registro. Esta opção visa facilita a interpretação dos dados em trabalhos de sísmica de refração, quando a definição exata do tempo da primeira chegada das ondas é vital.

Um cursor que pode ser movido pelo teclado do computador permite localizar pontos importantes no sismograma, e também o tempo correspondente pode ser visualizado. Esta função objetiva facilitar a interpretação prévia, ainda no campo, dos eventos de interesse.

O formato de gravação do arquivo em disco obedece a um padrão compatível com o sistema de processamento sísmico Proseis, desenvolvido por Talos Five Computer Software, no entanto, facilmente adaptável para qualquer outro padrâo desejado, uma vez que a diferenca entre os formatos utilizados pelos diversos sistemas de processamento está na ordenação das informaçōes, e não no conteúdo delas.

\section{TESTES DO SISTEMA COMPLE- TO}

Após concluidos os testes em cada parte do sistema, foram efetuados testes do sistema completo, tanto em laboratório como no campo, tendo apresentado comportamento bastante satisfatório.

O sistema vem sendo utilizado em diversos trabalhos para diversas áreas de pesquisas (p. ex. Taioli et al., 1993 e Taioli \& Dourado, 1994). Uma seção experimental obtida em área metropolitana da cidade de São Paulo é apresentada na Figura 12, onde é possível observar claramente o contacto do solo de alteração com o embasamento cristalino a cerca de $45 \mathrm{~m}$, que equivale, para a área, profindidade da ordem de 8 metros.

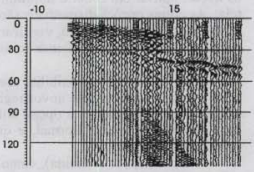

Figura 12 - Seção experimental executada com o sistema desenvolvido, em área urbana.

Posterior evoluçăo do sistema permitiu a implementação de sistema de fonte e sensor triortogonal para a execu- 
ção de ensaios entre furos (crosshole) efetuando-se pequenas alteraçōes no programa de controle. Esta versão do instrumento também vem sendo utilizada para pesquisas das caracteristicas dinâmicas dos maciços terrosos e rochosos na regiåo da Bacia Sedimentar de São Paulo, como subsídio à engenharia de fundaçōes (Décourt et al., 1994).

\section{COMENTÁRIOS E CONCLUSŌES}

O sistema (mecânica, eletrônica e programa de controle) aqui proposto foi projetado e construido para que fosse o mais econômico possivel, $\mathrm{e}$ atendesse às necessidades básicas e mais comuns da maioria dos geofísicos envolvidos em trabalhos sísmicos aplicados à engenharia, meio ambiente e hidrogeologia. Não pretende ser um sistema comercial, porém, pode ser de grande aplicaçảo a Universidades e Institutos de Pesquisas graças à sua arquitetura aberta e relativa simplicidade nas soluçōes adotadas. Deve-se ressaltar que se trata de um sistema básico, onde qualquer alteração $\mathrm{e}$ aperfeiçoamento se torna possivel em cada bloco ou módulo independentemente, sem necessidade de modificações do sistema como um todo. Esta característica, conseguida com a arquitetura adotada, deverá permitir uma continua evolução do sistema, e pode ser utilizada para o desenvolvimento de outros instrumentos geofísicos.

\section{AGRADECIMENTOS}

O autor deseja expressar os sinceros agradecimentos ao Prof. Nelson Ellert do IG-USP, Prof. J.Greenhouse e ao Sr. G.Schneider da University of Waterloo, aos colegas Nicolau Palm, Vilmondes Ribeiro e Dr. J.C.Dourado pelas valiosas discussões. Aos Srs. Joaquim Ireno e José P.Burato pelo apoio nos trabalhos de laboratório e campo. Ao Prof. Francisco Yukio Hiodo do
IAG-USP pela revisảo crítica do manuscrito. Finalmente, ao CEPAS - Centro de Pesquisas de Águas Subterrâneas pelos recursos para usinagem dos acelerômetros.

\section{REFERÊNCIAS BIBLIOGRÁFICAS}

AL-SADI, H.N. (1980) Seismic exploration: technique and processing. Cambridge, Birkhauser, Verlag. 215 p.

ANSTEY, N.A. (1981) Seismic prospecting instruments: signal characteristics and instrument specifications. Berlin, Gebrueder Borntraeger, v.1, 154p.

BENSON, R.C. (1993) Geophysical techniques for subsurface site characterization. In: DANIEL, D.E. (ed.) Geotechnical practice for waste disposal. London, Chapman, p.311357.

BORM, G.W. (1978) Methods from exploration seismology: reflection, refraction and borehole prospecting. In: BORM, G.W. (ed.) Rock dynamics and geophysical aspects, Rotterdam, Balkema, v.3, p.87-114.

BORN, W.T. (1960) A review of geophysical instrumentation. Geophysics, v.25, n.1, p.77-91.

COFFEEN, J.A. (1978) Seismic exploration fundamentals. Oklahoma, PPC Books, 277p.

DÉCOURT, L.; NIYAMA, S.; CAMPOS, G.C.; TAIOLI, F.; DOURADO, J.C. (1995) Experimental behavior of a piled footing. In: CONGRESSO PANAMERICANO DE MECÂNICA DOS SOLOS E ENGENHARIA DE FUNDAÇÕES, 10 ., Guadalajara, 1995. Anais. Guadalajara, IAMSFE, v.2, p.1083-1094.

DOBRIN, M.B. (1981) Introduction to Geophysical Prospecting. 3.ed. Auckland, McGraw-Hill, 630p.

EVENDEN, B.S.; PIEUCHOT, M. (1984) Seismic Prospecting In- 
struments. 2.ed. Berlin, Gebruder Borntraeger, v.1. (Geoexploration Monographs, Series 1), 158p.

FAII, J.P.; GRAU, G.; LAVERGNE, M. (1962) Couplage des sismographes avec le sol. Geophysical Prospecting. v. 10, n.2, p.128-147.

GEOSPACE. GS-30CT (1995) Close tolerance geophone. Catálogo de especificaçōes. USA. $2 \mathrm{p}$.

JAKOSKY, J.J. (1950) Exploration geophysies. 2.ed. California, Trija, p.639-937.

JOHNSON, D.E.; HILBURN, J.L. (1975) Rapid practical designs of active filters. New York, John Wiley \& Sons, 264p.

KEPPNER, G. (1991) Ludger Mintrop. The Leading Edge of Exploration. v.10, n.9, p.21-28.

KLAASSEN, K.B.; VAN PEPPEN, J.C.L. (1983) Electronic acceleration-sensitive geophone for seismic prospecting. Geophysical Prospecting, v. 31, p. $457-480$.

KNAPP, R.W.; STEEPLES, D.W. (1986a) High resolution commondepth-point seismic reflection profiling: instrumentation. Geophysics, v. 51, n.2, p. 276-282.

KNAPP, R.W.; STEEPLES, D.W. (1986b) High resolution commondepth-point seismic reflection profiling: field acquisition parameter design. Geophysics, v.51, n.2, p.283294.

LAMER, A. Couplage sol-geophone. Geophysical Prospecting, v.18, n.2, p.300-319.

LEPPER, C.M. (1981) Guidelines for selecting seismic detectors for highresolution applications. RI. Bureau of Mines. Report of Investigation, n. $8599,37 \mathrm{p}$.

NEIDELL, N.S. (1982) Following the selsmic pulse to the reflecting bed and back: selsmic wave propagation in Earth. In: APPLIED GEOPHYSICS FOR GEOLOGISTS, 10 ,
Houston, 1982. Annual Course. Houston. University of Houston Geology Foundation, p.1-94.

PARASNIS, D.S. (1986) Principles of Applied Geophysics. 4.ed., New York, Chapman and Hall, 402p.

PIEUCHOT, M. (1984) Seismic Instrumentation. In: HELBIG, K. TREITEL, S. (eds.) Handbook of geophysical exploration. London, Geophysical Press, v.2, p. 375.

PULLAN, S.E.; MAC AULAY, H.A. (1987) An in-hole shotgun source for engineering seismic surveys. Geophysics, v. 52 , n. 7 , p.985-996.

ROBINSON, E.S.; CORUH, C. (1988) Basic Exploration Geophysics. New York, John Wiley \& Sons. 562 p.

SEGUIN, M.K. (1971) La géophysique et les propriétés des roches. Québec, Les Presses de L'Université LAVAL, p. 445-1358.

SERRIDGE, M.; LICHT, T.R. (1986) Piezoelectric accelerometer and vibration preamplifiers: theory and application handbook. Denmark, Brüel and Kjaer. 151p.

SHARMA, P.V. (1986) Geophysical Methods in Geology, 2.ed. New York, Elsevier, 442p.

SHERIFF, R.E. (1979) A first course in geophysical exploration and interpretation. Boston, International Human Resources Development Corporation. 313p.

SHERIFF, R.E. (1989) Geophysical Methods. New Jersey, Prentice Hall, 605 p.

STERNBERG, B.K. (1995) Status and future growth of near-surface geophysics. The Leading Edge, v.14, n.4, p. 230-269.

TAIOLI, F. (1992) Desenvolvimento e testes de sistema sismográfico de alta resolução. São Paulo, $119 \mathrm{p}$. (Tese - Doutorado) - Instituto de Geociências, Universidade de São Paulo. 
TAIOLI, F.; RIBEIRO, V. (1990a). Sistema automático de aquisição de dados para medida de permeabilidade de maciços rochosos "in-situ". In: SIMPÓSIO SOBRE INSTRUMENTAÇÃO EM GEOTECNIA/ SINGEO 90. Anais. Rio de Janeiro, ABMS, p.311-317.

TAIOLI, F.; RIBEIRO, V. (1990b) Sistema automático para ensaio de determinação do tensor de permeabilidade. In: CONGRESSO BRASILEIRO DE GEOLOGIA DE ENGENHARLA, 6., Salvador, 1990. Anais. Salvador, ABGE, v.1, p.115-120.

TAIOLI, F.; DOURADO, J.C.; CORDEIRO, R.P. (1993) Determinação de matacões por sísmica de reflexão de alta resolução. In: CONGRESSO BRASILEIRO DE GEOLOGIA DE ENGENHARIA, 7., Poços de Caldas, 1993. Anais. Poços de Caldas, ABGE, v.1, p.219-226.

TAIOLI, F.; DOURADO, J.C. (1994) Geofisica aplicada a problemas urbanos: um novo desafio. In: CONGRESSO BRASILEIRO DE GEOLOGIA, 38., Camboriú, 1994. Boletim de Resumos Expandidos. Camburiú, SBG, v.2, p.16-17.

TELFORD, W.M.; GELDART, L.P.; SHERIFF, R.E. (1990) Applied Geophysics. 2.ed., Cambridge, Cambridge University, 770p.

TEXAS INSTRUMENTS. (1982) DFS V - drawings manual. Houston, Texas Instruments, p.2.9.

WASHBURN, H.; WILEY, H. (1941) The effect of the seismometer on its response characteristics. Geophysics, v.6, n.1, p.116-131.

WHITE, J.E.; PRESS, F. (1960) Geophysical research and progress in exploration. Geophysies, v.25, n.1, p.168-180.

WOLFF, E.A.; MERCANTI, E.P. (Eds.) (1974) Geoscience Instrumentation. New York, John Wiley \& Sons, 819p.

F.Taioli - Instituto de Geociências, Universidade de Såo Paulo, Caixa Postal 11.348, CEP 05422-970, São Paulo, SP, Brasil. 